\title{
Delayed decrement of the nerve impulse propagation during induced limb ischaemia in chronic hepatic failure
}

\author{
VIGGO KAMP NIELSEN ${ }^{1}$ AND TROELS KARDEL \\ From the Department of Clinical Neurophysiology, The Department of Neurology, \\ and the Department of Surgery S, University Hospital, Gentofte, Denmark
}

SYNOPSIS Sensory thresholds for vibrations and electrical shocks and the sensory nerve conduction velocity (median nerve) were measured during 30 minutes of induced limb ischaemia in 10 normal subjects and 15 patients with chronic hepatic failure. Sensory action potentials were recorded simultaneously at the wrist and the elbow. Seven patients (group A) had a normal perception time for vibrations. As in normal subjects, the potential amplitude decreased from the onset of ischaemia, more pronounced at the elbow than at the wrist, and the conduction velocity became reduced more rapidly in the wrist-elbow than in the first digit-wrist segment, indicating impulse propagation with decrement. Eight patients (group B) had a markedly prolonged perception time for vibrations. The reduction of potential amplitude and conduction velocity was considerably delayed, and the impulse propagation showed no evidence of decrement for 20-25 minutes of ischaemia. A rise of the threshold. for electrical shocks was absent or very delayed. As in the two other groups, the rise was abrupt and occurred at the moment when the potential below the occluding cuff (elbow) was extinct. In both groups of patients there was an increasing temporal dispersion of action potentials and in patient group A a desynchronization of the late phase of the potential took place early in the course of ischaemia. Differences between groups A and B could not be predicted from the pre-ischaemic nerve conduction data.

Peripheral nerve function during ischaemia is sometimes better preserved in patients with certain metabolic disorders than in normal subjects. This was shown in diabetic patients for vibratory perception (Steiness, 1959), and has later been demonstrated for touch and pain as well (Gregersen, 1968). The phenomenon has also been demonstrated in patients with uraemia (Christensen and Ørskov, 1969), chronic hepatic failure (Seneviratne and Peiris, 1970), and hypercalcaemia (Gregersen and Pilgaard, 1971). In a previous study, we reported that vibratory perception was abnormally preserved during ischaemia in 18 out of 33 patients $(54 \%)$ with severe hepatic failure (Kardel and Nielsen, 1974). The present paper further extends this study by an

1 Address for correspondence: Dr V. Kamp Nielsen, Neuromedical dept N, Rigshospitalet, Blegdamsvej 9, 2100, Copenhagen $\varnothing$, Denmark.

(Accepted 1 May 1975.) electrophysiological examination of the phenomenon of ischaemia resistance in selected groups of patients from the material. ${ }^{2}$

In normal human nerves subjected to ischaemia we have observed the following sequence of electrophysiological events (Nielsen and Kardel, 1974). The sensory conduction velocity and the amplitude of the sensory action potential showed a considerable decrease most pronounced in the nerve segment just below the occluding cuff, without any significant alteration in the sensory threshold. At the moment when the action potential just below the occluding cuff became extinct, the threshold increased abruptly. The conduction velocity in the ischaemic nerve decreased as a function of the distance from the

2 Part of this study was presented at the 20th Congress of Scandinavian Neurologists, Oslo 1972 (Nielsen and Kardel, 1972) and at The EEG Society Joint Meeting, Edinburgh 1974 (Nielsen and Kardel, 1975). 966 
point of stimulation and of the duration of ischaemia. This is compatible with the concept that the nervous impulse becomes propagated with decrement after a certain impairment of the axon membrane function (Szpilman and Luchsinger, 1881; Lorente de Nó and Condouris, 1959).

It is the object of the present investigation to reconsider these findings in patients with chronic hepatic failure with and without an increased resistance of nerve function during ischaemia. We have examined the propagation of the same nerve action potential over two adjacent segments of the ischaemic nerve, which is a prerequisite for the demonstration of decremental conduction. Our study indicates that the greater resistance of the nerve function during ischaemia is a temporary phenomenon due to delay of a normal sequence of electrophysiological events, and hence does not suggest physiological mechanisms different from those in normal nerves.

\section{METHODS}

PATIENTS Fifteen patients, six females and nine males, 43 to 64 years of age (mean $=53$ years), with cirrhosis of the liver verified by biopsy and chronic hepatic failure were selected from a larger patient material described previously (Kardel and Nielsen, 1974). The patients were divided into two groups according to their vibratory perception threshold curves recorded on the pulp of the index finger during a $30 \mathrm{~min}$ period of limb ischaemia.

Group A Seven patients showed a normal response -that is, rapidly increasing vibratory perception threshold after 10-15 minutes of ischaemia. The ischaemic perception time (IPT) averaged 19 minutes (range: 12-24 minutes). IPT was defined as the time (min) when the perception of a vibration stimulus of maximal strength $(50 \mathrm{~V}$ to an electromagnetically driven vibrator) was extinct.

Group $B$ Eight patients showed an abnormal threshold curve during ischaemia. Six patients still perceived vibrations at the end of the 30 minute test period, and the rise of the threshold was also considerably delayed in two, IPT being $28 \mathrm{~min}$ in both.

The two groups showed the same age and sex distribution (Table 1). Chronic alcoholism was the suspected cause of cirrhosis in four patients of group $A$ and five of group B, and a surgical portacaval shunt had been made in five patients in each group. There was no difference between the mean values of
TABLE 1

CLINICAL DATA (MEAN \pm SEM)

\begin{tabular}{|c|c|c|c|}
\hline & \multirow{2}{*}{$\begin{array}{l}\text { Normal } \\
\text { limit }\end{array}$} & \multicolumn{2}{|c|}{ Cirrhosis patients } \\
\hline & & $\begin{array}{c}\text { Group } A \\
(N=7)\end{array}$ & $\begin{array}{c}\text { Group } B \\
(N=8)\end{array}$ \\
\hline $\begin{array}{l}\text { Age (yr) } \\
\text { Sex (males/females) }\end{array}$ & & $\begin{array}{c}54 \pm 2.3 \\
4 / 3\end{array}$ & $\begin{array}{c}53 \pm 2.3 \\
5 / 3\end{array}$ \\
\hline \multicolumn{4}{|l|}{ Liver function } \\
\hline capacity $(\mathrm{mg} / \mathrm{min})$ & $>350$ & $273 \pm 20$ & $256 \pm 11$ \\
\hline Bilirubin (mg/dl) & $<1.2$ & $1.2 \pm 0.07$ & $2.2 \pm 0.54$ \\
\hline \multicolumn{4}{|l|}{ Prothrombin-proconvertin } \\
\hline$(\%)$ & $70-120$ & $61 \pm 9.5$ & $53 \pm 5.0$ \\
\hline Albumin (g/dl) & $3.9-4.7$ & $3.5 \pm 0.25$ & $4.0 \pm 0.13$ \\
\hline Gamma-globulin (g/dl) & $0.7-1.3$ & $2.0 \pm 0.26$ & $1.8 \pm 0.31$ \\
\hline \multicolumn{4}{|l|}{ Oral glucose tolerance } \\
\hline \multicolumn{3}{|l|}{ Fasting blood glucose } & $111 \pm 21$ \\
\hline $\begin{array}{l}\text { Blood glucose, } 120 \mathrm{~min} \\
(\mathrm{mg} / \mathrm{dl})\end{array}$ & $<115$ & $98 \pm 13$ & $192 \pm 30^{*}$ \\
\hline
\end{tabular}

* P (group A vs group B) $<0.02$.

routine liver function tests, the serum concentrations of bilirubin, prothrombin-proconvertin, albumin, and gamma globulin. The mean galactose elimination capacity, measuring the functioning liver mass, was of the same order in the two groups and was moderately to severely reduced in all patients, ranging from 223 to $344 \mathrm{mg}$ per $\min$ (lower normal limit = $350 \mathrm{mg}$ per min; Tygstrup, 1964). An oral glucose tolerance test showed a 'diabetic' response in six of the patients in group B, while all patients in group A had a normal or intermediate response according to the criteria of Megyesi et al. (1967). None received treatment with insulin.

NORMAL CONTROLS Ten normal subjects, five females and five males, 40-71 years of age (mean = 52 years) served as controls. The IPT for vibrations ranged from 15-21 minutes, on average 18 minutes.

ELECTROPHYSIOLOGICAL EXAMINATION The sensory conduction velocity in the median nerve was examined as described by Buchthal and Rosenfalck (1966). A detailed description of the ischaemia test has been given in a previous report (Nielsen and Kardel, 1974). In brief, sensory action potentials were recorded simultaneously at the wrist and the elbow with needle electrodes after supramaximal stimuli (duration $0.2 \mathrm{~ms}$ ) to sensory nerve fibres distally on digit 1 . The near-nerve electrode, insulated except for a $3 \mathrm{~mm}$ bare tip, was inserted close to the nerve (motor threshold stimulus strength lower than $1.0 \mathrm{~mA}$ ). The remote electrode was placed at a transversal distance of $30 \mathrm{~mm}$. The action potentials were displayed on a 
TABLE 2

PRE-ISCHAEMIC NERVE CONDUCTION DATA (MEAN \pm SEM)

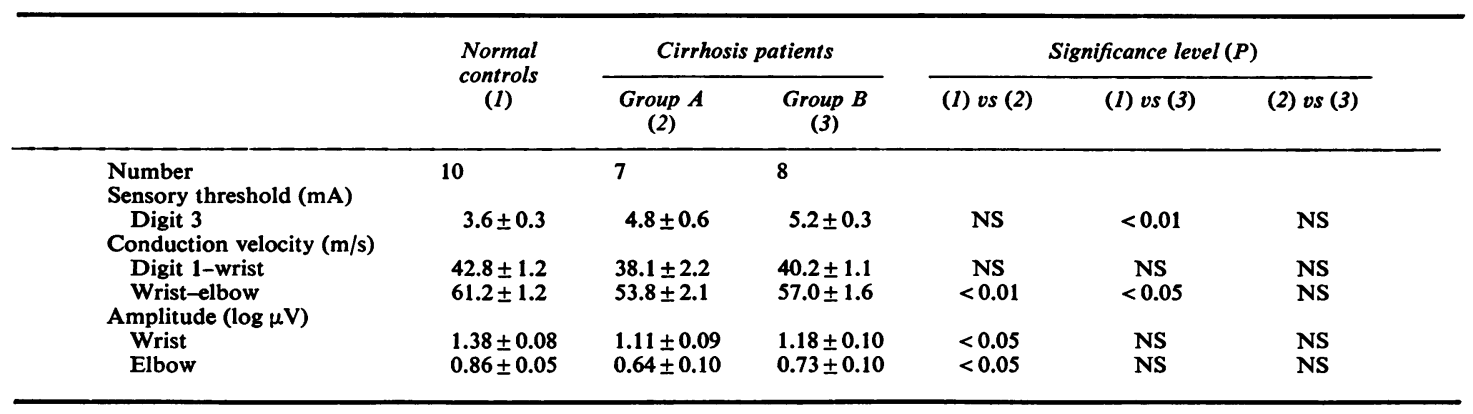

three-channel electromyograph (DISA). The sensory threshold to electrical shocks (duration $=0.2 \mathrm{~ms}$ ) was measured distally on digit 3 . The skin temperature at the wrist and elbow was kept at about $35^{\circ} \mathrm{C}$ by external heating.

Recordings were made before and every two to three minutes during a $\mathbf{3 0}$ minute period of limb ischaemia produced by the inflation of a pneumatic cuff around the upper arm, placed $5 \mathrm{~cm}$ proximally to the elbow electrode. The pressure was maintained at at least $50 \mathrm{mmHg}$ above the systolic blood pressure. Changes in the nerve conduction parameters, conduction velocities $(\mathrm{m} / \mathrm{s})$, and potential amplitude $(\mu \mathrm{V})$ were expressed as the percentage value of the pre-ischaemic observation. The mean percentage value was calculated in each group for every period of three minutes. Differences between patient groups and normal controls were tested with a two-tailed Student's $t$ test.

\section{RESULTS}

The pre-ischaemic nerve conduction data are summarized in Table 2. The average sensory threshold (digit 3) was elevated in patient group B. The average conduction velocity in the digit 1-wrist segment did not differ from the normal in either of the two patient groups, while a significant reduction was present in the wrist-elbow segment in both groups. The average amplitude of the evoked action potentials was slightly reduced in patient group $A(P<0.05)$. Before ischaemia there was no significant difference between patient groups A and B for any of the five electrophysiological variables.

ISCHAEMIA Sensory threshold (Fig. 1) In normal subjects the sensory threshold was stationary for about 15 minutes after which an abrupt tenfold increase was seen. A 71 year old male had a more gradual increase after 23 minutes of ischaemia. He was the oldest person examined. The sensory threshold in group A showed a

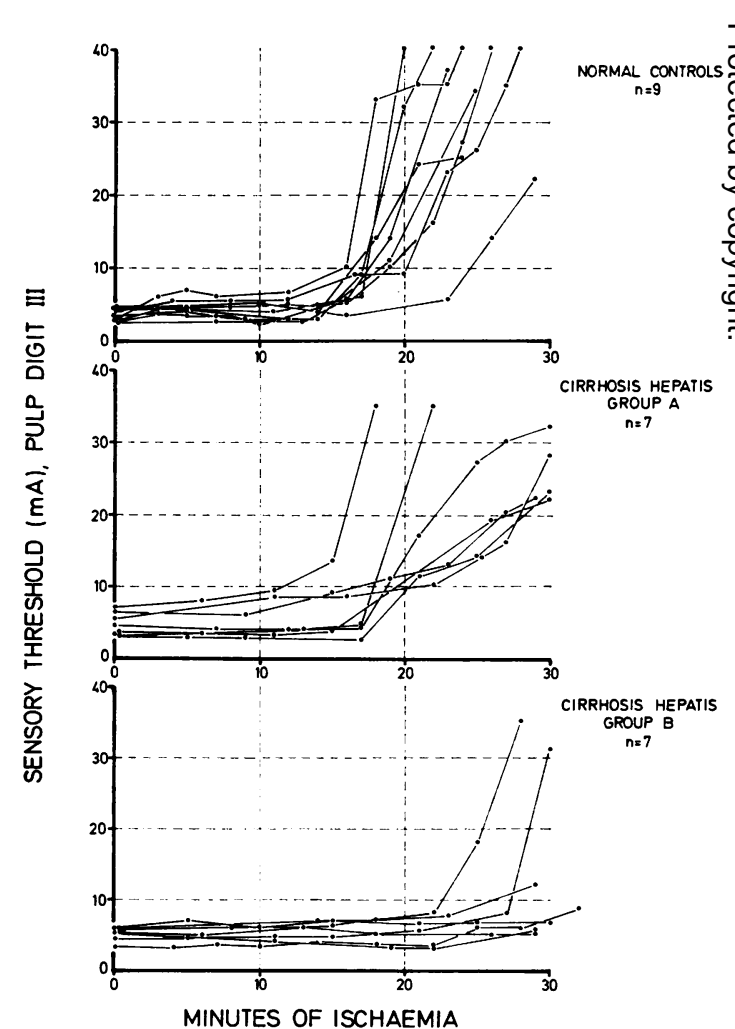

FIG. 1 Sensory thresholds to electrical shocks during ischaemia. The distal phalanx of digit 3 was stimulated via ring electrodes. 
similar course, but the rising phase was usually less abrupt. However, in all patients the threshold reached twice the pre-ischaemic value within 20 minutes of ischaemia. Only two patients in group B (female 64 years, and male 53 years old) showed a distinct rise of the threshold after 22 and 27 minutes of ischaemia. Both had ischaemic perception times for vibrations of 28 minutes. In the other six patients with preserved vibration sense after 30 minutes of ischaemia, the sensory threshold to electrical shocks was either unchanged or only slightly increased at the end of the 30 minute test period.

Sensory conduction velocity (Fig. 2) In normal

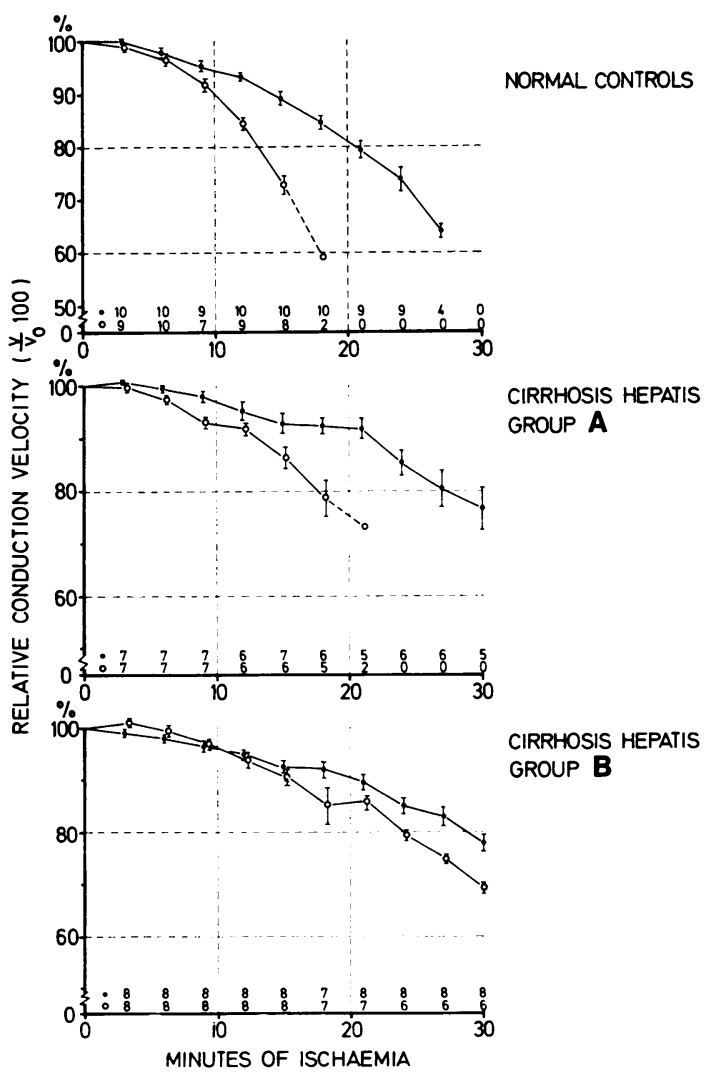

FIG. 2 Changes in the sensory conduction velocity (median nerve) during ischaemia, $\bigcirc=$ digit 1 -wrist segment, $\bigcirc=$ wrist-elbow segment. Each point indicates the average percentage deviation from the preischaemic value $\pm S E M$. Figures above the abscissa indicate the number of observations. subjects the conduction velocity consistently showed a more rapid decrease in the wristelbow segment than in the digit-wrist segment. The average relative conduction velocities of the two segments diverged significantly after nine minutes of ischaemia. After 15-18 minutes the nerve conduction was blocked in the proximal segment, while the conduction velocity could still be measured in the distal segment for another 10 minutes of ischaemia or more. This phenomenon has previously been analysed in further detail (Nielsen and Kardel, 1974).

A similar conduction pattern was seen in the patients in group $A$, but the decrease in the conduction velocity as a function of the duration of ischaemia was more delayed. The average relative conduction velocities of the two segments diverged significantly after 12 minutes, and the nerve conduction in the wrist-elbow segment was blocked after 18-21 minutes (Fig. 4).

The conduction pattern in group B differed markedly from that in the two other groups. In group B a very delayed reduction of the conduction velocity in the distal and proximal nerve segments ran in parallel during the first 24 minutes of ischaemia. Then a growing and statistically significant difference was demonstrable. However, after 30 minutes of ischaemia the nerve conduction was still preserved in the proximal segment in six of eight patients, and in the distal segment in all eight patients. For any given duration of ischaemia the conduction velocity was less reduced than in the two other groups.

Nerve conduction measurements were continued for 52 minutes of ischaemia in one of these patients (female aged 50 years). Table 3 shows the parallel slowing of the conduction velocity in the distal and proximal segments. The difference between the relative velocities of the two segments did not exceed $10 \%$ until after 34 minutes, and even after 52 minutes of ischaemia a nerve impulse could be conducted through the entire length of the ischaemic nerve (Fig. 4). At this time the conduction velocity had decreased to $53 \%$ and $33 \%$ of the pre-ischaemic value in the distal and proximal segments respectively. After the blood circulation in the arm had been restored, the nerve conduction recovered more slowly than usually. Therefore, after 37 minutes the electrode positions were carefully marked, and on the following day a reexamination showed 


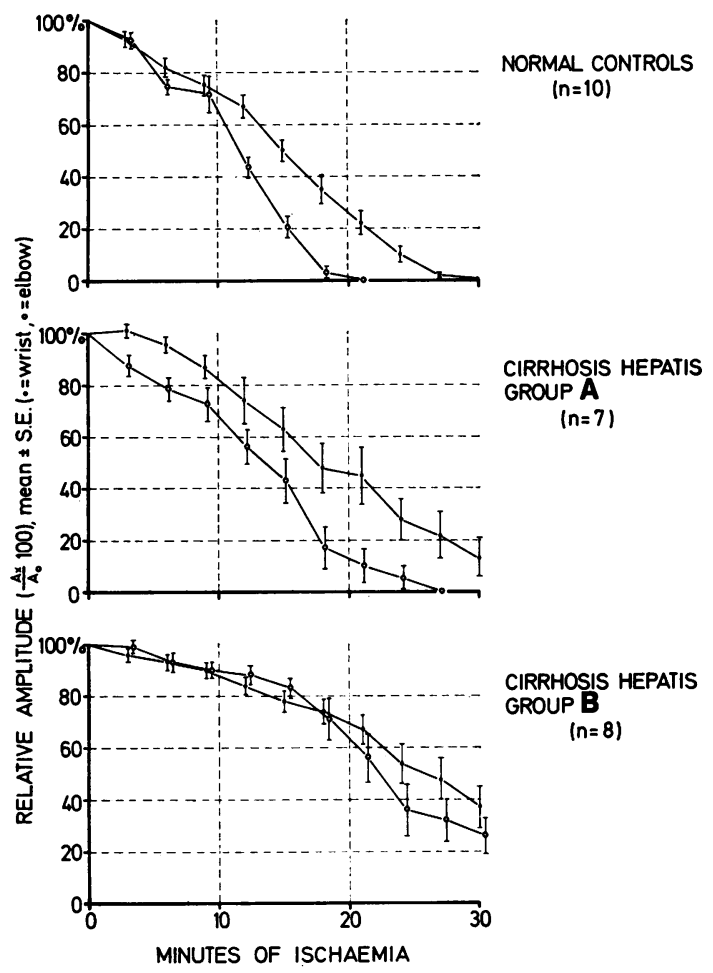

FIG. 3 Changes in the potential amplitude during ischaemia. Sensory action potentials after supramaximal stimulation of digit 1 were recorded through needle electrodes placed near the nerve at the wrist (O), and the elbow (O). Each point indicates the average percentage deviation from the pre-ischaemic value $\pm S E M$.

conduction velocities close to the pre-ischaemic values and within the limits of intraindividual variation (Nielsen, 1973).

Amplitude (Fig. 3) In normal subjects the decrease in the amplitude of the action potentials was considerably faster at the elbow than at the wrist. Action potentials disappeared at the elbow after 15-18 minutes and at the wrist after 24-27 minutes of ischaemia.

The potential amplitude in group A tended to decrease at a slower rate than in normal subjects. Thus, in two patients the elbow potential did not disappear until 21 and 24 minutes after onset of ischaemia, and the wrist potential was still present in five patients after 30 minutes.

In group B the decrease in amplitude was not
TABLE 3

CONDUCTION VELOCITIES AND POTENTIAL AMPLITUDES BEFORE, DURING, AND AFTER 52 MIN OF LIMB ISCHAEMIA (EMG 315, FEMALE, 50 YR)

\begin{tabular}{|c|c|c|c|c|c|}
\hline \multirow{2}{*}{$\begin{array}{l}\text { Time } \\
0 \text { min }\end{array}$} & \multicolumn{2}{|c|}{$\begin{array}{l}\text { Conduction velocity } \\
\qquad(\mathrm{m} / \mathrm{s}(\%))\end{array}$} & \multicolumn{3}{|c|}{$\begin{array}{l}\text { Amplitude } \\
(\mu V(\%))\end{array}$} \\
\hline & $\begin{array}{l}\text { Digit 1-wrist } \\
45(100)\end{array}$ & $\begin{array}{l}\text { Wrist-elbow } \\
67(100)\end{array}$ & $\begin{array}{c}\text { Wrist } \\
38(100)\end{array}$ & $\begin{array}{c}\text { Elbow } \\
16(100)\end{array}$ & \\
\hline \multicolumn{6}{|l|}{ Ischaemia } \\
\hline $2 \mathrm{~min}$ & $45(100)$ & $67(100)$ & 36 (95) & $15(94)$ & \\
\hline 4 & $44(98)$ & 66 (98) & 34 (90) & $14(88)$ & \\
\hline 6 & 44 (98) & 64 (96) & 32 (84) & 13 (81) & \\
\hline 9 & 44 (98) & 63 (94) & 32 (84) & 12 (75) & \\
\hline 10 & 44 (98) & 63 (94) & 30 (79) & $12(75)$ & \\
\hline 12 & 43 (96) & 62 (92) & 28 (74) & 11 (69) & \\
\hline 14 & 42 (93) & 61 (91) & 28 (74) & 10 (63) & \\
\hline 16 & 41 (91) & $58 \quad(87)$ & $26(68)$ & 10 (63) & \\
\hline 18 & 41 (91) & 58 (87) & $26(68)$ & 9 (56) & \\
\hline 20 & 40 (89) & 57 (85) & 24 (63) & 9 (56) & \\
\hline 22 & 39 (87) & $55(82)$ & 22 (58) & 7 (44) & \\
\hline 24 & 38 (84) & 54 (81) & 20 & 6 (38) & \\
\hline 26 & 37 (82) & 50 (75) & 17 (45) & 6 (38) & \\
\hline 28 & 35 (78) & 50 (75) & 16 (42) & 5 (31) & \\
\hline 30 & 35 (78) & 47 (70) & 14 (37) & 4 (25) & \\
\hline 32 & 34 (76) & 45 (67) & - & - & \\
\hline 34 & 32 (71) & 40 (60) & - & - & \\
\hline 36 & 31 (69) & 38 (57) & 11 (29) & 3 (19) & \\
\hline 38 & 30 (67) & 35 (52) & $10(26)$ & 3 (19) & \\
\hline 40 & 30 (67) & 34 (51) & $10(26)$ & 2 (13) & \\
\hline 44 & 28 (62) & 30 (45) & 10 (26) & 2 (13) & $\bar{\pi}$ \\
\hline 47 & $26(58)$ & 28 (42) & 8 (21) & 2 (13) & O \\
\hline 52 & 24 (53) & 22 (33) & 7 (18) & 2 (13) & (D) \\
\hline \multicolumn{6}{|c|}{ Blood flow reestablished } \\
\hline $56 \mathrm{~min}$ & $28(62)$ & - & 11 (29) & - & O \\
\hline 58 & 30 (67) & 31 (46) & 17 (45) & - & \\
\hline 60 & 30 (67) & $34(51)$ & 18 (47) & $6(38)$ & กุ \\
\hline 62 & 32 (71) & 36 (54) & 19 (50) & 6 (38) & 응 \\
\hline 68 & 35 (78) & 37 (55) & 18 (47) & $6(38)$ & $\leq$ \\
\hline 75 & $36(80)$ & 39 (58) & 17 (45) & $5(31)$ & $\overline{\bar{\theta}}$ \\
\hline 80 & 37 (82) & 41 (61) & $16(42)$ & 5 (31) & ? \\
\hline 91 & 39 (87) & 45 (67) & 17 (45) & 5 (31) & \\
\hline $24 \mathrm{hr}$ & 42 (93) & 66 (98) & 36 (95) & 10 (63) & \\
\hline
\end{tabular}

only much more delayed than in normal subjects, but like the conduction velocity the degree $\stackrel{\square}{\triangle}$ of reduction was of the same order of magnitude for action potentials recorded at the wrist and at the elbow. After 20 minutes of ischaemia the mean values were systematically lower at the elbow than at the wrist, but the difference did not become statistically significant during the 30 minute test period. After 30 minutes, action potentials were still present in all eight patients at the wrist and in six patients at the elbow, averaging $37 \%$ and $26 \%$ of the pre-ischaemic values respectively.

In the patient examined for 52 minutes of $\frac{D}{2}$ ischaemia (Table 3), the reduction in amplitude was almost linear for about 30 minutes, with a slightly greater slope at the elbow than at the 


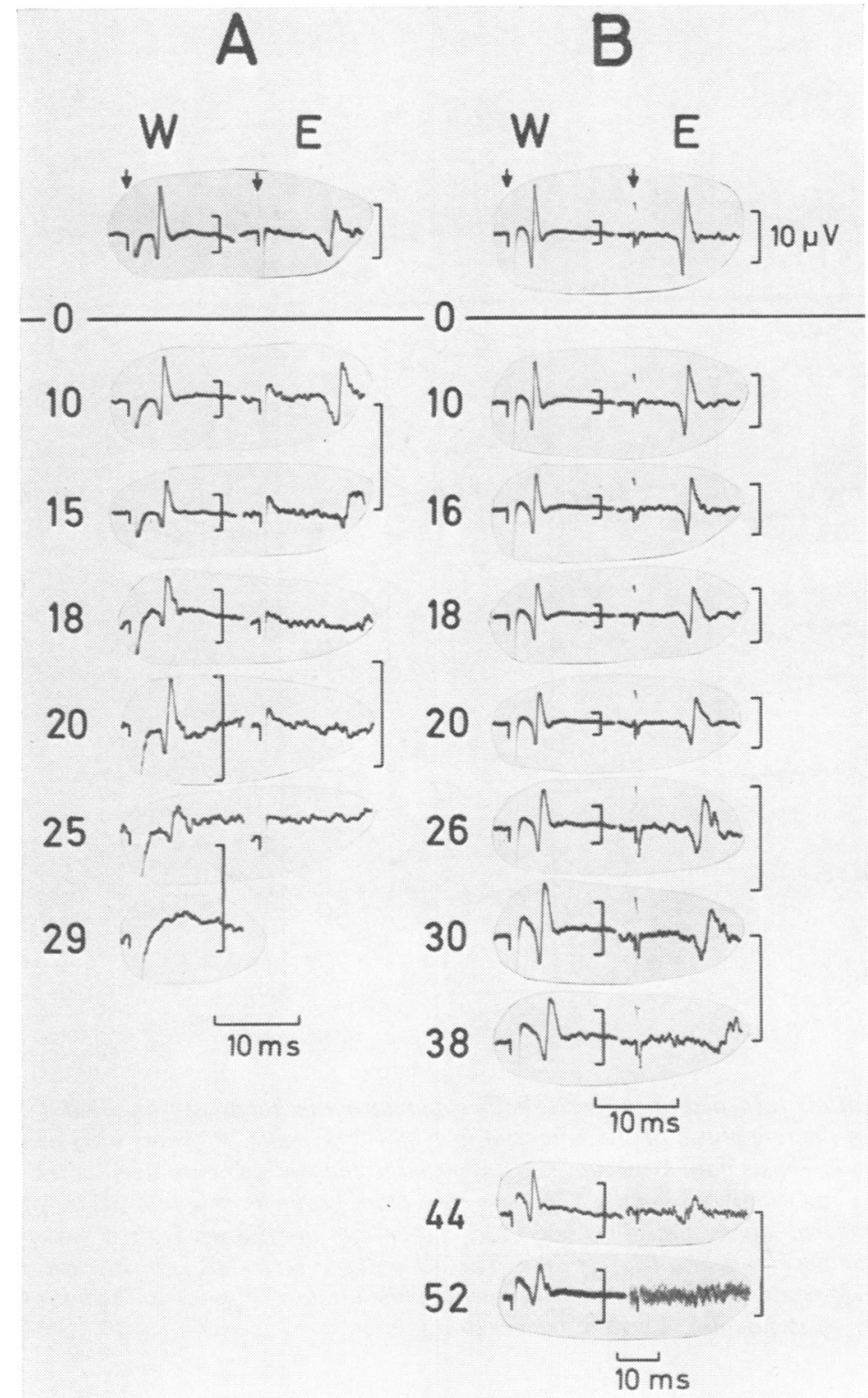

FIG. 4 Sensory action potentials recorded at the wrist $(\mathrm{W})$ and the elbow (E) (stim. digit 1) before and during limb ischaemia in patients with chronic hepatic failure. A: EMG 191, male, 45 years old, with a normal ischaemia perception time for vibrations. B: EMG 315, female, 50 years old, with a greatly prolonged ischaemic perception time for vibrations. Figures to the left of the action potentials indicate minutes of ischaemia. Vertical bars $=10 \mu \mathrm{V}$. The sweep velocity was $0.5 \mathrm{~ms} / \mathrm{mm}$ with the exception of the two last recordings in patient $B$, where the sweep velocity was $1 \mathrm{~ms} / \mathrm{mm}$ (44 and $52 \mathrm{~min}$ ). All potentials are recorded single sweep except the last recordings in patient $B(52 \mathrm{~min})$, where 20 potentials were superimposed in order to discriminate the highly polyphasic potential at the elbow.

wrist. After 52 minutes a polyphasic action potential of $2 \mu \mathrm{V}$ could be recorded at the elbow (Fig. 4). The amplitudes of wrist and elbow potentials 37 minutes after reestablishment of the blood circulation were restored to only $45 \%$ and $31 \%$ of the pre-ischaemic value, mainly due to desynchronization. At reexamination on the following day, both potentials were well syn- chronized but the elbow potential still had an amplitude of only $63 \%$ of the pre-ischaemic value, possibly due to a difference in the position of the near-nerve electrode.

Action potentials In an earlier paper we demonstrated that in normal subjects the sensory action potentials during ischaemia showed 


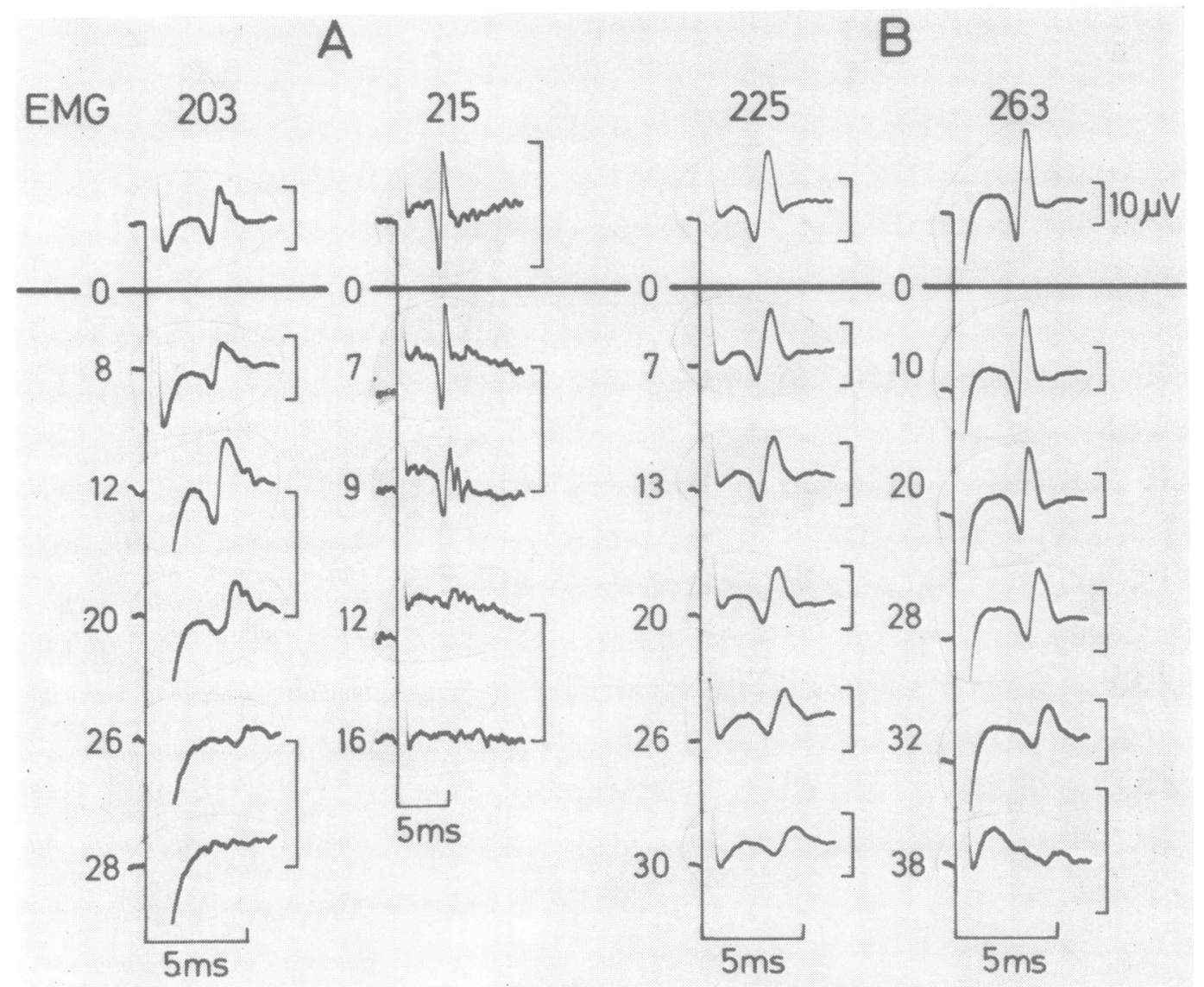

FIG. 5 Selected sensory action potentials recorded at the wrist before and during ischaemia (stim. digit 1). Note the gradual desynchronization of the late phase of the potential in EMG 203 (male, 57 years old) and 215 (male, 50 years old) from hepatic cirrhosis patient group $A$, as opposed to the well synchronized potentials in EMG 225 (female, 56 years old) and 263 (male, 53 years old) from group B. All four series of potentials showed an increasing temporal dispersion. In EMG 215, potentials are shown with a sweep velocity of $0.5 \mathrm{~ms} / \mathrm{mm}$ to illustrate the development of late peaks. The other three series are shown with a sweep velocity of $0.25 \mathrm{~ms} / \mathrm{mm}$, which was always used for measurement of the latency. Figures to the left of the action potentials indicate minutes of ischaemia. Vertical bars $=10 \mu \mathrm{V}$.

increased temporal dispersion, defined by the latency difference between the first and second positive peaks (Nielsen and Kardel, 1974). In patients with chronic hepatic failure a similar increased temporal dispersion was seen, more prominent in group A than in group B. However, in many patients, the second positive peak was ill defined, which precluded a numerical analysis of changes in temporal dispersion.

In group A a gradual desynchronization of the action potential was a characteristic feature. This was always seen in the late phase of the potential, while the first positive peak remained well synchronized during the major part of the $\frac{\rho}{3}$ ischaemic period. Desynchronization could be $\circ$ demonstrated already in action potentials re- $?$ corded at the wrist-that is, close to the point of stimulation. Similar observations, although not commented on, were made in our previous study in normal subjects. 


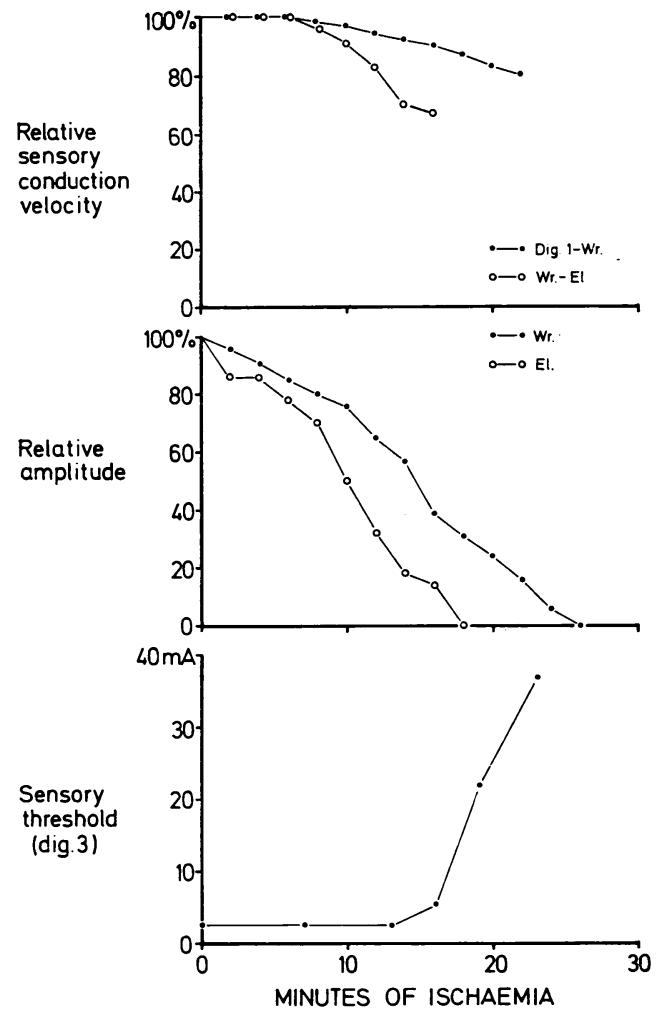

FIG. 6 Typical changes in the sensory conduction velocity in the median nerve, in the amplitude of action potentials, and in the sensory threshold to electrical shocks during ischaemia in a normal person (EMG 265, male 41 years old). The pre-ischaemic conduction velocities were $45.0 \mathrm{~m} / \mathrm{s}$ (digit 1-wrist) and $59.2 \mathrm{~m} / \mathrm{s}$ (wrist-elbow). The pre-ischaemic potential amplitudes were $46 \mu \mathrm{V}$ (wrist) and 11.2 $\mu V$ (elbow).

In group B action potentials remained well synchronized almost for 30 minutes of ischaemia. Thus, in action potentials recorded at the wrist, only a slow increase of the temporal dispersion was seen, while potentials recorded at the elbow most remote from the point of stimulation did show irregularities and separate peaks late in the course and mainly confined to the late phase of the potential. Typical action potentials recorded during ischaemia from patients of groups A and $B$ are shown in Figs 4 and 5.

Relationship between variables Figure 6 shows a typical example of simultaneous changes in the

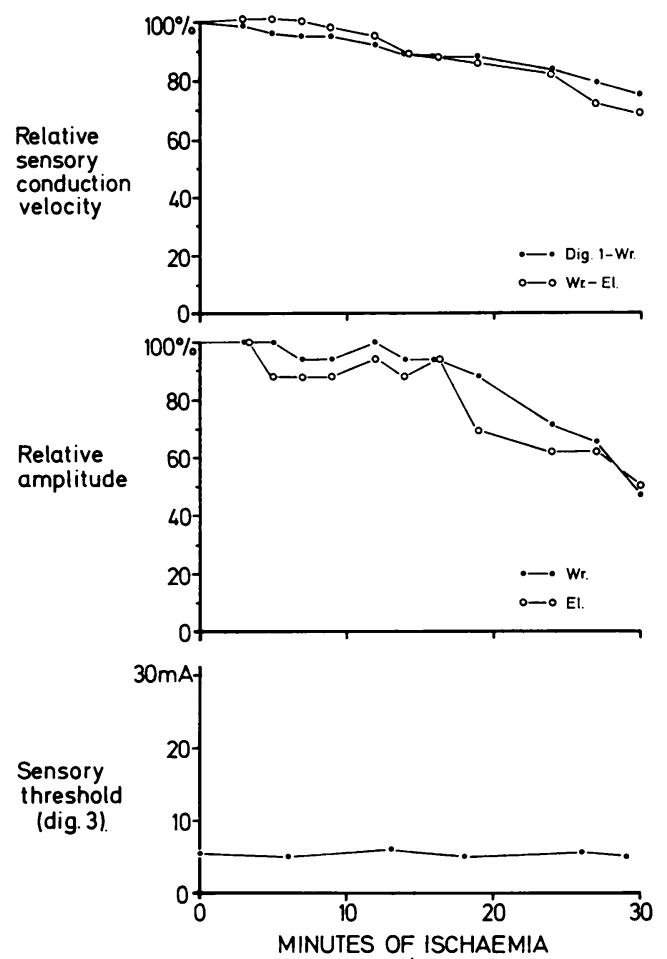

FIG. 7 Typical changes in the sensory conduction velocities in the median nerve, in the amplitude of action potentials, and in the sensory threshold to electrical shocks during ischaemia in a patient with chronic hepatic failure and with an abnormally preserved vibration sense during ischaemia (EMG 190, male 53 years old). The pre-ischaemic conduction velocities were $41.8 \mathrm{~m} / \mathrm{s}$ (digit 1-wrist) and $56.5 \mathrm{~m} / \mathrm{s}$ (wrist-elbow). The pre-ischaemic potential amplitudes were $13.6 \mu V$ (wrist) and $3.2 \mu V$ (elbow).

five electrophysiological parameters during ischaemia in a normal subject, representative also for patients in group A. Figure 7 shows the same parameters in a patient with increased ischaemia resistance from group B. In the normal subject, a reduction of the potential amplitude was soon followed by a slowing of the conduction velocity, most pronounced in the proximal segment. The sensory threshold was unaffected by a $30 \%$ reduction of the conduction velocity in the wristelbow segment and a decrease in the amplitude of more than $80 \%$ at the elbow. The rapid rise of the threshold coincided with the moment when the elbow potential disappeared. In the group B 
patient the potential amplitude at the wrist and elbow and the conduction velocity in distal and proximal segments decreased slowly and almost in parallel. The elbow potential was preserved throughout the test period and the sensory threshold remained practically unchanged.

\section{DISCUSSION}

The present study describes the conduction of the same action potential along an ischaemic nerve segment. The ischaemic nerve conduction pattern in group A was almost identical with that in normal subjects. This supports earlier observations that the conduction velocity decreases as a function of the duration of ischaemia and of the distance from the point of stimulation - that is, with decrement-and that the action potential becomes extinct at a gradually shorter distance from the point of stimulation, the longer the duration of ischaemia (Nielsen and Kardel, 1974). In group B, the slowing of the conduction velocity was greatly delayed during ischaemia, and during 20-25 minutes of ischaemia the decrease was of the same order in the distal and the proximal nerve segment. After that time, the conduction velocity in all patients became reduced at a faster rate in the wrist-elbow segment than in the digit-wrist segment with a significant difference between the average values after 24 minutes (see also Table 3).

As in normal subjects, patients in group A showed a clear reduction of the potential amplitude before any tendency to decremental conduction was seen. Since other factors (increased temporal dispersion, block of fibres, changes in the electrode position) can be disregarded during the early course of ischaemia (Nielsen and Kardel, 1974), we assume that the decrease in amplitude reflects a reduction of the single fibre membrane potential. The amplitude decreased much faster at the elbow than at the wrist from the onset of ischaemia. In contrast, patients in group B showed an almost parallel reduction at the two recording sites. Hence, it appears that the ability to maintain a non-decremental impulse propagation despite a reduction of the potential amplitude distinguishes patients of group B from patients of group $A$ and from normal subjects.
It has been argued that the decrement pheno- $\bar{z}$ menon might be due to a selective block of the fastest conducting fibres. However, the gradualo increase of the temporal dispersion of the potential already demonstrated in normal sub- 0 jects (Nielsen and Kardel, 1974), was also observed in both groups of patients in this study, $\frac{\overline{\mathrm{O}}}{\mathrm{T}}$. more pronounced in group A. This indicates that ischaemia results in a slowing of the conduction: velocity in fast as well as more slowly conducting $\stackrel{\overrightarrow{\vec{S}}}{+}$ fibres. Moreover, in persons demonstrating early읃 decrement (normal subjects and group A), a $\frac{\bar{c}}{\bar{n}}$ gradual desynchronization of the potential was $\frac{\bar{\sigma}}{\widehat{D}}$ early present even at the wrist. The interesting $\cong$ fact is that the desynchronization was virtually क confined to the late phase of the potential, which $\overrightarrow{0}$ represents the small-diameter slowly conducting $\overrightarrow{\vec{H}}$ myelinated fibres, while the initial phase repre- $\omega$ senting the large-diameter fast conducting fibres remained well synchronized even until late in the? ischaemia period. This challenges the classical concept that the large diameter fibres are the $\overrightarrow{0}$ fibres most susceptible to ischaemia (Gasser and Erlanger, 1929). On the contrary, one wou政 expect from a biophysical point of view that the small-diameter fibres would be affected first ared most severely. Inhibition of the outward Nad transport due to ischaemia would lead to a rise $\frac{\text { I }}{2}$ the intracellular $\mathrm{Na}^{+}$concentration and a de crease of the resting membrane potential which $\overrightarrow{0}$ would be greater the smaller the fibre diameter. of The selective desynchronization of the late phase of the action potential fits in with the latter concept. We acknowledge, however, that the method applied in this study does not allow a definitive $\frac{0}{8}$ conclusion as to the relative degree of involve- $\Omega$ ment of fast and slow fibres. This would presuppose an averaging of a large number of 3 potentials which is impossible during ischaemia due to the time factor. On the other hand, our data strongly indicate that the decrement phenomenon is not due to a selective block of the fast conducting, large-diameter fibres. The well- 3 known preservation of pain sensation after loss of tactile sensation might be due to other painconveying fibre types - for example, non-myelin- $ᄋ$ ated-exhibiting other characteristics during $\rightarrow$ ischaemia, which might be disclosed after a block of the presynaptic inhibition of pain impulses in $N$ the spinal cord due to block of myelinated fibres (Melzack and Wall, 1965). Action potentials in 
our studies represent myelinated fibres only above 4-6 $\mu \mathrm{m}$ in diameter.

The effect of ischaemia on the sensory threshold for electrical shocks was substantially the same in patients of groups $\mathrm{A}$ and $\mathrm{B}$ and in normal subjects. The threshold was unchanged as long as an action potential could be recorded at the elbow about $5 \mathrm{~cm}$ distal to the cuff, increasing abruptly at the moment when the elbow potential was extinguished. This is further evidence that the deciding factor for the preservation of the threshold appears to be whether or not the threshold action potential is at all able to reach and depolarize the unaffected axon membrane proximal to the cuff. As a consequence, the unchanged sensory threshold in patient group B is merely due to the extended preservation of a sufficiently large action potential at the elbow, and is thus a temporary phenomenon only.

As shown in Table 2, the two groups of patients $A$ and $B$, could not be distinguished on the basis of the pre-ischaemic nerve conduction data despite the marked differences in the ischaemic nerve conduction patterns. Similarly, differences in the pre-ischaemic values between normal subjects and group A patients were not reflected by any significant difference in the ischaemia nerve conduction pattern, and as seen from the dispersion of data, several patients in group B had pre-ischaemic nerve conduction values well within the normal range. This indicates that the ischaemic response is a separate parameter of peripheral nerve function, supplementing other electrophysiological informations.

Several explanations for the increased ischaemia resistance have been suggested. (1) Abnormalities in glucose metabolism have been suspected as a common denominator for diabetes, uraemia, and hepatic failure. During ischaemia, energy might be provided from anaerobic glycolytic pathways, as suggested by the antagonistic effect of glucose on the decrease of the nerve potential in frog nerves during anoxia (Shanes, 1951). In fact, an abnormal oral glucose tolerance test in group B constituted the only difference from group A among the biochemical factors tested. However, previous studies have shown that an abnormal glucose tolerance was not a precondition for increased ischaemia resistance, neither in uraemia (Christensen and Ørskov, 1969) nor in chronic hepatic failure
(Seneviratne and Peiris, 1970; Kardel and Nielsen, 1974). (2) The increased ischaemia resistance in patients with hypercalcaemia has been referred to a calcium-ion dependent decrease of the sodium permeability through the ischaemic axon membrane (Gregersen and Pilgaard, 1971). This mechanism is hardly relevant in patients with chronic hepatic failure where the ionized calcium concentration is normal or slightly decreased (Moore, 1971). (3) It has been suggested that a shorter internode distance, resulting from segmental demyelination, might facilitate the saltatory impulse propagation when the resting membrane potential becomes reduced during ischaemia (Christensen and Ørskov, 1969). There are several objections to this suggestion (see also Seneviratne and Peiris, 1970). Thus, in the hypertrophic type of Charcot-Marie-Tooth's disease with severe demyelination, patients do not exhibit the phenomenon of increased ischaemia resistance (Gregersen and Pilgaard, 1971), and patients with the carpal tunnel compression syndrome even show increased susceptibility to ischaemia with a shortening of the resistance period (Gilliatt and Wilson, 1954; Fullerton, 1963). In our study, the increased resistance is mainly due to the preserved conduction in the proximal segment of the ischaemic nerve, which is least demyelinized, if at all. (4) Recently, Seneviratne and colleagues $(1970,1972,1974)$ have presented data from diabetic patients and rats suggesting an increased cation permeability of the periaxonal diffusion barrier in the nodal gap of Ranvier. This was assumed to counteract the build-up of an extra-membrane potassium concentration, hence delaying a decrease of the membrane potential. Although changes in a periaxonal barrier substance have not been described in patients with hepatic failure, the uniform decrease of the potential amplitude at the wrist and the elbow in our study might be compatible with this concept. This could be the case if the observed amplitude reduction were mainly due to an accumulation of $\mathrm{Na}^{+}$in the axoplasm caused by ischaemic inhibition of the outward $\mathrm{Na}^{+}$transport.

Although the pathophysiological background for the increased ischaemia resistance is still obscure, our results may add to the clarification of this discussion. It is evident that these patients 
show an ability to maintain an axon membrane polarization for a longer period of time than normal subjects, as shown by the preserved action potential at the elbow in this study. Moreover, it appears that the sequence of electrophysiological events in patients with increased ischaemia resistance after some delay is very similar to that in normal subjects. This does not suggest any major difference in the physicochemical mechanisms. The initial reduction of the potential amplitude in group $B$ can be ascribed to the growing temporal dispersion of the potential, as shown in Figs 4 and 5. Thus, it is possible that the single fibre membrane potential could be relatively well preserved, which might explain why the conduction velocity in these patients is initially slowed without decrement.

\section{REFERENCES}

Buchthal, F., and Rosenfalck, A. (1966). Evoked action potentials and conduction velocity in human sensory nerves. Brain Research, 3, 1-122.

Christensen, N. J., and Ørskov, H. (1969). Vibratory perception during ischaemia in uraemic patients with mild carbohydrate intolerance. Journal of Neurology, Neurosurgery, and Psychiatry, 32, 519-524.

Fullerton, P. M. (1963). The effect of ischaemia on nerve conduction in the carpal tunnel syndrome. Journal of Neurology, Neurosurgery, and Psychiatry, 26, 385-397.

Gasser, H. S., and Erlanger, J. (1929). The role of fiber size in the establishment of a nerve block by pressure or cocaine. American Journal of Physiology, 88, 581-591.

Gilliatt, R. W., and Wilson, T. G. (1954). Ischaemic sensory loss in patients with peripheral nerve lesions. Journal of Neurology, Neurosurgery, and Psychiatry, 17, 104-114.

Gregersen, G. (1968). A study of the peripheral nerves in diabetic subjects during ischaemia. Journal of Neurology, Neurosurgery, and Psychiatry, 31, 175-181.

Gregersen, G., and Pilgaard, S. (1971). The effect of ischaemia on vibration sense in hypo- and hypercalcaemia and in demyelinated nerves. Acta Neurologica Scandinavica, 47, 71-79.
Kardel, T., and Nielsen, V. K. (1974). Hepatic neuropathy. A clinical and electrophysiological study. Acta Neurologica Scandinavica, 50, 513-526.

Lorente de Nó, R., and Condouris, G. A. (1959). Decremental conduction in peripheral nerve. Integration of stimuli in the neuron. Proceedings of the National Academy of Sciences of the United States of America, 45, 592-617.

Megyesi, C., Samols, E., and Marks, V. (1967). Glucose tolerance and diabetes in chronic liver disease. Lancet, 2, 1051-1055.

Melzack, R., and Wall, P. D. (1965). Pain mechanism: a new theory. Science, 150, 971-979.

Moore, E. W. (1971). Studies with ion exchange calcium $\overline{\bar{ज}}$ electrodes. 3. The state of serum calcium in patients with cirrhosis. Gastroenterology, 60, 43-53.

Nielsen, V. K. (1973). Sensory and motor nerve conduction in the median nerve in normal subjects. Acta Medica Scandinavica, 194, 435-443.

Nielsen, V. K., and Kardel, T. (1972). Sensory nerve conduction during ischaemia in normal subjects and patients with chronic liver disease. Acta Neurologica Scandinavica, 48, suppl. 51, 467-469.

Nielsen, V. K., and Kardel, T. (1974). Decremental conduction in normal human nerves subjected to ischaemia? Acta Physiologica Scandinavica, 92, 249-262.

Nielsen, V. K., and Kardel, T. (1975). Increased resistance of the nerve function during ischaemia in chronic hepatic failure. Electroencephalography and Clinical Neurophysiology, 38, 213.

Seneviratne, K. N., and Peiris, O. A. (1970). Peripheral nerv function in chronic liver disease. Journal of Neurologe Neurosurgery, and Psychiatry, 33, 609-614.

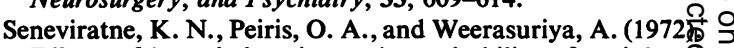
Effects of hyperkalaemia on the excitability of peripheraf nerve. Journal of Neurology, Neurosurgery, and Psychiatr 35, 149-155.

Seneviratne, K. N., and Weerasuriya, A. (1974). Nodal ga substance in diabetic nerve. Journal of Neurology, Neuros $\mathbb{D}$ surgery, and Psychiatry, 37, 502-513.

Shanes, A. M. (1951). Factors in nerve functioning. Federaf tion Proceedings, 10, 611-621.

Steiness, I. (1959). Vibratory perception in diabetics during arrested blood flow to the limb. Acta Medica Scandinavica, 163, 195-205.

Szpilman, J., and Luchsinger, B. (1881). Zur Beziehung von Leitungs und Erregungsvermögen der Nervenfaser. Archiv für die gesamte Physiologie, 24, 347-357.

Tygstrup, N. (1964). The galactose elimination capacity in control subjects and in patients with cirrhosis of the liver. Acta Medica Scandinavica, 175, 281-289. 